\title{
Constrained Registration for Motion Compensation in Atrial Fibrillation Ablation Procedures
}

\author{
Alexander Brost, Andreas Wimmer, Rui Liao, Felix Bourier, Martin Koch, Norbert Strobel, Klaus Kurzidim, \\ and Joachim Hornegger, Member, IEEE,
}

\begin{abstract}
Fluoroscopic overlay images rendered from preoperative volumetric data can provide additional anatomical details to guide physicians during catheter ablation procedures for treatment of atrial fibrillation (AFib). As these overlay images are often compromised by cardiac and respiratory motion, motion compensation methods are needed to keep the overlay images in sync with the fluoroscopic images. So far, these approaches have either required simultaneous biplane imaging for 3-D motion compensation, or in case of monoplane X-ray imaging, provided only a limited 2-D functionality. To overcome the downsides of the previously suggested methods, we propose an approach that facilitates a full 3-D motion compensation even if only monoplane $X$-ray images are available. To this end, we use a training phase that employs a biplane sequence to establish a patient specific motion model. Afterwards, a constrained model-based 2-D/3-D registration method is used to track a circumferential mapping catheter. This device is commonly used for AFib catheter ablation procedures. Based on the experiments on real patient data, we found that our constrained monoplane 2-D/3-D registration outperformed the unconstrained counterpart and yielded an average 2-D tracking error of $0.6 \mathrm{~mm}$ and an average 3-D tracking error of $1.6 \mathrm{~mm}$. The unconstrained 2-D/3-D registration technique yielded a similar 2-D performance, but the 3-D tracking error increased to $3.2 \mathrm{~mm}$ mostly due to wrongly estimated 3-D motion components in X-ray view direction. Compared to the conventional 2-D monoplane method, the proposed method provides a more seamless workflow by removing the need for catheter model re-initialization otherwise required when the $\mathrm{C}$-arm view orientation changes. In addition, the proposed method can be straightforwardly combined with the previously introduced biplane motion compensation technique to obtain a good trade-off between accuracy and radiation dose reduction.
\end{abstract}

Index Terms-2-D/3-D Registration, Ablation, Atrial Fibrillation, Electrophysiology, Motion Compensation

\section{INTRODUCTION}

A TRIAL fibrillation (AFib) is the most common arrhythmia. It leads to an increased stroke risk for patients [1]. Since the first treatment approaches using radio-frequency ablations by Haiissaguerre et al. [2], this method has now become an accepted treatment option, in particular, when drug therapy fails [3], [4], [5]. Catheter ablation procedures are

A. Brost, A. Wimmer, M. Koch and J. Hornegger are with the Pattern Recognition Lab, Friedrich-Alexander-University of Erlangen-Nuremberg, Erlangen, Germany, e-mail: Alexander.Brost@informatik.uni-erlangen.de.

N. Strobel is with Siemens AG.

R. Liao is with Siemens Corporate Research.

F. Bourier and K. Kurzidim are with Klinik für Herzrhythmusstörungen, Krankenhaus Barmherzige Brüder, Regensburg, Germany.

Copyright (c) 2012 IEEE. Personal use of this material is permitted. However, permission to use this material for any other purposes must be obtained from the IEEE by sending a request to pubs-permissions@ieee.org. performed in electrophysiology (EP) labs usually equipped with modern C-arm X-ray systems. These devices often provide 3 -D tomographic imaging to facilitate inter-procedural 3-D soft-tissue imaging [6], [7], [8], [9]. Electro-anatomic mapping systems are also available to visualize the catheter position in 3-D within a registered 3-D data set [10], [11], [12], [13]. While they promise to save X-ray dose, they add effort and cost to the procedure. In addition, mapping systems are virtual reality systems, and they do not allow for instant confirmation of catheter positions under real-time X-ray. In some instances, they may even be off with respect to the underlying anatomy [14].

Augmented fluoroscopy, overlaying 2-D renderings obtained from either CT, MR, or C-arm CT 3-D data sets onto live fluoroscopic images, can facilitate more precise realtime catheter navigation and also reduce X-ray dose [15], [16], [17]. Unfortunately, catheter navigation under augmented fluoroscopy is compromised by cardiac and respiratory motion. A first approach to tackle this problem by providing a motion compensated overlay was proposed in [18], [19]. It involved tracking of commonly used circumferential mapping (CFM) catheters. As atrial fibrillation therapy takes place in the vicinity of the circumferential mapping catheter, tracking of this catheter can be assumed to reliably capture the motion of the relevant treatment region if the device has been firmly positioned. Fortunately, we can count on the physicians to provide a stable wall contact, as it is in their best interest. Otherwise complete isolation of the pulmonary veins (PVs) may fail due to undetected residual PV-atrial electrical connections. Our previously proposed method involved a 3-D model of the catheter and applied an unconstrained 2-D/3-D registration approach to align the catheter model to biplane fluoroscopy images. An initial registration is performed manually to align the 3-D data to 2-D fluoroscopy with contrast injection showing the target organ. Once the 3-D overlay moves in sync with live fluoroscopic images, catheters can be guided to anatomical structures otherwise not visible under fluoroscopy with more confidence. Another approach to register a pre-operative data to biplane fluoroscopy had been proposed before [20]. We extended this approach to AFib ablation procedures where the catheter used for registration is not placed in a single vessel. Furthermore, we use the catheter to perform an automatic registration over time to perform motion compensation.

A yet different approach for monoplane fluoroscopic imaging was introduced in [21]. There, the catheter was tracked only in 2-D and the overlay image was moved accordingly, i.e., the projection of the pre-operative 3 -D data set was shifted on 
the live fluoroscopic images to be in sync with the cardiac and respiratory motion, observed by localizing the 2-D mapping catheter.

In EP labs equipped with biplane $\mathrm{C}$-arm systems, often only one image plane is used at a time to reduce the radiation exposure to the patient. In this case, the methods suggested in [18] is not applicable. The 2-D method described in [21] is not ideal either, as it requires re-initialization of the catheter model if the $\mathrm{C}$-arm projection geometry changes during the intervention. Even though model re-initialization is not a very time consuming step, it does interrupt the workflow. To overcome the shortcomings of both methods, we propose to apply constrained 2-D/3-D registration to perform motion compensation. Our approach is based on a 3-D catheter model and an estimated patient-specific motion model learned during a training phase. Set-up of the 3-D catheter model requires only a single biplane X-ray image and follows the method explained in [18], [19]. A similar approach for coronary interventions had been proposed in [22]. The training phase estimates the motion of the CFM catheter at the PV using a biplane sequence in which the mapping catheter is tracked using an unconstrained 2-D/3-D registration. The principal motion axis is determined from the trajectory established during the tracking training phase. This axis is considered a patient-specific motion model. Since the main axis of the motion by itself is not sufficient to provide a good search space for a constrained registration, another axis is needed. We decided use a vector perpendicular to the viewing direction and the main axis, because the search region for the constrained registration can then be reduced to a 2-D search space, spanned by the principal axis and a vector parallel to the image plane. This allows us to not only track motion that is parallel to the image plane, but also to capture some depth information with respect to the pre-acquired motion model. Our constrained approach can be simply stated as dimension reduction of the search space from 3-D to 2-D.

The main contribution of our paper is the estimation of a patient-specific motion model for the circumferential mapping catheter positioned at the pulmonary vein considered for ablation to obtain pulmonary vein isolation (PVI). The motion model is used to generate a 2-D search space which is used for a constrained 2-D/3-D motion compensation.

The paper is organized as follows. In the first section, the 3-D catheter model set-up required for motion compensation is briefly summarized. This catheter model is used for the model-based 2-D/3-D registration, with the catheter model representing the 3-D information. The second section focuses on the image processing techniques and catheter segmentation. A distance transform of the catheter segmentation is used as cost function. At the same time, it is the basis for the registration step. Thereafter, the patient-specific motion model is presented. This model is estimated during a training phase in which the circumferential mapping catheter is tracked using biplane X-ray imaging. The training is performed on a biplane sequence to obtain the main motion axis. In the fourth section, the constrained 2-D/3-D registration based on the motion model is introduced. Thanks to the estimated motion model, motion compensation can be constrained to two dimensions.

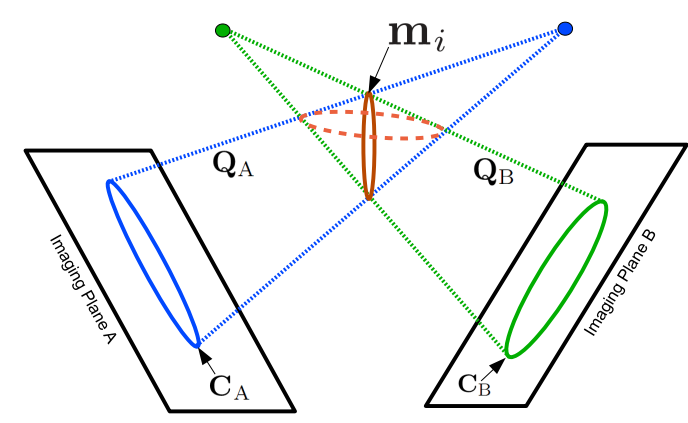

Fig. 1. A sketch of the 3-D elliptical catheter model generation.

The first is the main axis of the observed motion field, and the second is perpendicular to the viewing direction and the main motion axis. In the last section, we discuss our results and consider future directions.

\section{3-D Elliptical CATHeter Model}

In this section, we summarize the 3-D catheter model generation from two views using manually selected points as input. This method was proposed in [19]. The method requires that points are set on the catheter displayed in a single biplane $\mathrm{X}$-ray acquisition. Beyond that, this method does not put any restrictions on the operator, i.e., no special parts of the catheter need to be selected. Put differently, 2-D points $\mathbf{p}_{\mathrm{A}}, \mathbf{p}_{\mathrm{B}} \in \mathbb{R}^{2}$ are selected on the elliptically-shaped part of the catheter in each image plane. The two image planes are denoted by $\mathrm{A}$ and $\mathrm{B}$. Two-dimensional ellipses $\mathbf{C}_{\mathrm{A}}, \mathbf{C}_{\mathrm{B}} \in \mathbb{R}^{3 \times 3}$ in the image planes are then fitted to these points using the algorithm in [23]. If the 2-D point were on a perfect ellipse, the matrices would satisfy the following equations

$$
\begin{aligned}
& \widetilde{\mathbf{p}}_{\mathrm{A}}^{T} \mathbf{C}_{\mathrm{A}} \widetilde{\mathbf{p}}_{\mathrm{A}}=0 \\
& \widetilde{\mathbf{p}}_{\mathrm{B}}^{T} \mathbf{C}_{\mathrm{B}} \widetilde{\mathbf{p}}_{\mathrm{B}}=0
\end{aligned}
$$

with the 2-D points $\mathbf{p}_{\mathrm{A}}$ and $\mathbf{p}_{\mathrm{B}}$ in homogeneous coordinates as $\widetilde{\mathbf{p}}_{\mathrm{A}}=\left(\mathbf{p}_{\mathrm{A}}^{T}, 1\right)$ and $\widetilde{\mathbf{p}}_{\mathrm{B}}=\left(\mathbf{p}_{\mathrm{B}}^{T}, 1\right)$. For ellipse fitting, at least six points are required. The method in [23] performs ellipse fitting in a least-squares sense if more than six points are provided. A constraint is used to ensure that the solutions for $\mathbf{C}_{\mathrm{A}}$ and $\mathbf{C}_{B}$ are elliptical [24], [23]. After the ellipses in the image planes are fitted, ellipse reconstruction in 3-D is performed using the method proposed in [25]. To this end, two 3-D cones $\mathbf{Q}_{\mathrm{A}}, \mathbf{Q}_{\mathrm{B}} \in \mathbb{R}^{4 \times 4}$ are computed using the projection matrices $\mathbf{P}_{\mathrm{A}}, \mathbf{P}_{\mathrm{B}} \in \mathbb{R}^{3 \times 4}$. The cones are spanned from the cameras optical centers to the ellipses on the image planes, see Fig. 1 . Details on C-arm projection geometry are given in [26]. Two cones are computed as quadrics by

$$
\begin{aligned}
& \mathbf{Q}_{\mathrm{A}}=\mathbf{P}_{\mathrm{A}}^{T} \mathbf{C}_{\mathrm{A}} \mathbf{P}_{\mathrm{A}} \\
& \mathbf{Q}_{\mathrm{B}}=\mathbf{P}_{\mathrm{B}}^{T} \mathbf{C}_{\mathrm{B}} \mathbf{P}_{\mathrm{B}} .
\end{aligned}
$$

The quadrics $\mathbf{Q}_{\mathrm{A}}, \mathbf{Q}_{\mathrm{B}} \in \mathbb{R}^{4 \times 4}$ are of rank 3. Every intersection of a plane with one of the cones yields a valid solution of an ellipse in 3-D when projected onto the respective image plane. Given two cones, there are two possible solutions by calculating the two intersecting planes of the two 3-D 
cones [25]. Intersecting the 3-D cones with the two 3-D planes yields two possible solutions. The more circular solution of the two ellipses in 3-D is assumed to be the correct one [19], given the assumption that the pulmonary veins are tubularly shaped and that the circumferential mapping catheter is attached to the PV firmly. The 3-D model is denoted as $\mathbf{m}_{i}=\left(m_{i, x}, m_{i, y}, m_{i, z}, 1\right)^{T} \in \mathbb{R}^{4}$ in homogeneous coordinates with $i \in[1, N]$ and $N$ is the number of model points. During our experiments, we found that $N=50$ is sufficient to achieve good results. Increasing the number of model points did not lead to improved results. This step needs to be performed only once. For a sketch of the reconstruction process, see Fig. 1

\section{IMAGE PROCESSING}

Once the 3-D model of the catheter has been generated, only monoplane fluoroscopic imaging is needed for the remaining tasks of motion estimation and compensation. Compared to the monoplane method in [21], [27], the newly proposed method does not require manual re-initialization of the catheter model when $\mathrm{C}$-arm angulation changes, minimizing user interaction during interventions.

\section{A. Catheter Segmentation}

In order to segment the catheter in the fluoroscopic images, we first crop the input image with $1024 \times 1024$ pixels to a region-of-interest $(\mathrm{ROI})$ with $400 \times 400$ pixels around the projected center of the catheter model. The ROI size of $400 \times 400$ was chosen to make sure that we can always find the circumferential mapping catheter in all of our images. Using a different ROI size, or to be more specific, a higher search range would affect the runtime only. In the first frame, the position is known from the initialization step, and in all the subsequent frames, the tracking result from the previous frame is used.

The catheter segmentation method needs to be reliable and fast to allow motion estimation and compensation at the frame rate of the EP procedure, which typically ranges between 1 to 10 frames per second. We have decided on a combination of Haar-like features and a cascade of boosted classifiers. AdaBoost [28] is generally regarded as one of the best offthe-shelf classifiers [29], and in combination with Haar-like features has proven extremely successful, for example in the field of real-time face detection [30]. Haar-like features calculate various patterns of intensity differences. Some features detect edges, whereas others focus on line-like structures and are useful for detecting thin objects such as the catheter. Examples of feature prototypes are given in Fig. 2(a) Actual features are generated by shifting and scaling the prototypes within a predefined window. Thereby, contextual information around the center pixel is considered, which is important to differentiate between the catheter and background structures. Haar-like features can be calculated efficiently through integral images [30].

Even for moderate window sizes, the number of generated features is large, about 40,000 for the $15 \times 15$ pixel window we have chosen. The most suitable features for discriminating between catheter and background are selected by the AdaBoost algorithm [28] and integrated into a classifier. The idea is to combine several weak classifiers, which only have to be slightly better than chance, to form a strong classifier. In the simplest case, a weak classifier amounts to a single feature and threshold. During training, weak classifiers are repeatedly evaluated on samples of a training set where the catheter has been annotated. The classifier minimizing the classification error is added to a linear combination of weak classifiers until the overall error is below the desired threshold. After each iteration, the importance of individual samples is re-weighted to put more emphasis on misclassified samples for the next evaluation. A concise description is given in [29].

Instead of single features and thresholds, we use classification and regression trees (CARTs) [31] as weak classifiers. A CART is a small tree of fixed size, as illustrated in Fig. 2(b) At each node, a threshold associated with a feature partitions the feature space. Through this decomposition, flexibility is increased and objects with complex feature distributions can be handled. The value at the leaf represents the response of the classifier and indicates either catheter (positive) or background (negative). The number of five splits (and thus six leaves) in a CART was found to perform best in our experiments. When using a smaller number, the discriminative power of the tree may be too small, which in turn requires a large number of trees for each stage. On the other hand, when using a larger number of splits, the tree may over fit to the training data, limiting its applicability to the unseen test data.

Several strong classifiers, each consisting of weighted combinations of CARTs, are organized into a cascade [30] with $N$ stages, see Fig. 2(c). At each stage of the cascade, a sample is either rejected or passed on to the next stage. Only if the sample is accepted at the final stage, it is assumed to belong to the object. Since many background pixels can be rejected at an early stage and since their number is large compared to the number of catheter pixels, this approaches reduces the computational cost when applying the cascade for catheter segmentation. During training, the focus is on maintaining a high true positive rate while successively reducing the false positive rate, either by adding more weak classifiers to a stage or by adding an entirely new stage. We aim at a true positive rate of at least $99.5 \%$ and a false positive rate of not more than $50 \%$ per stage. A high true positive rate is required as a positive sample has to pass all the way down the cascade and must be accepted also by the last stage. When proceeding from stage to stage, a true positive rate of 0.995 per stage results in an overall true positive rate of $0.995^{N}$ for $N$ stages. In case of $N=4$ stages, the expected true positive rate of the whole cascade is about $98 \%$. For smaller true positive rates, the overall rate of the cascade would quickly decline. By setting the false positive rate to 0.5 per stage, we demand that each stage of the cascade halves the remaining number of false positives. In case of $N=4$ stages, the expected overall false positive rate is $0.5^{N}=6.25 \%$ With a higher false positive rate, more stages would be required to achieve an overall low false positive rate, whereas for a lower rate per stage, less stages but a more powerful strong classifier per stage would have to be trained. A gold-standard segmentation 


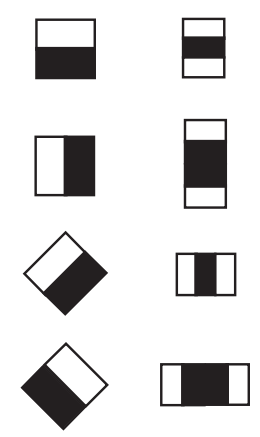

(a) Feature Types

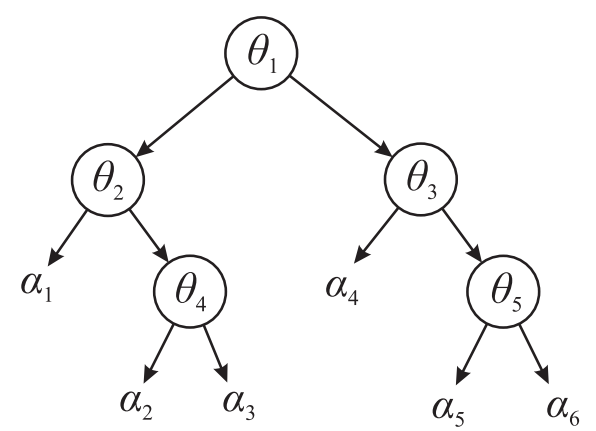

(b) Classification and Regression Tree (CART)

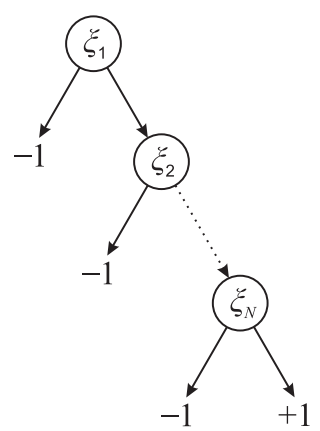

(c) Cascade

Fig. 2. Feature types and classifier structure for catheter segmentation: (a) Several Haar filter examples used for feature extraction; (b) Example of classification and regression tree (CART) with five feature nodes $\theta_{1}, \ldots, \theta_{5}$ and six leaves $\alpha_{1}, \ldots, \alpha_{6} ;$ (c) Classifier cascade consisting of $N$ stages with strong classifiers $\xi_{1}, \ldots \xi_{N}$. Each strong classifier $\xi_{i}$ consists of a linear combination of weak classifiers, here CARTs.

of the catheter was used for training.

\section{B. Image Post-Processing}

Different types of circumferential mapping catheters may be used for EP ablation purpose. They can differ in the number of electrodes as well as the thickness of the catheter. To ensure that our method can deal with a wide variety of catheters, we perform some post-processing on the segmentation output from the learning-based catheter classifier. First, the catheter segmentation results are smoothed by a median filter. In our experiments, a kernel size of $5 \times 5$ yielded the best results. Second, the smoothed segmentation is thinned using the method in [32], so that the thickness of the catheter no longer needs to be taken into consideration in the following registration step. Third, in order to obtain a smooth representation of the circumferential mapping catheter for subsequent efficient registration, the distance transform for the skeletonized image is calculated [33]. The distance transform of an image calculates for every background pixel the distance to the closest object pixel. The resulting image is denoted as $\mathbf{I}_{\mathrm{DT}}$. The image processing steps are summarized in Fig. 3

\section{Patient-Specific Motion Compensation}

In the following section, we present our method for the generation of a patient specific motion model. A short biplane sequence is used to generate 3-D samples of the position of the circumferential mapping catheter recorded during a training phase. The principal axis derived from the sample positions is taken as main direction of PV motion. The motion model should be acquired in the same state (heart rate in sinus rhythm, arrhythmia) that will be present during the application of the motion model. In the next step, we apply a constrained model-based 2-D/3-D registration to track the circumferential mapping catheter in 3-D using monoplane fluoroscopy. To this end, the motion model estimated during the training phase limits the allowed motion to two directions. The first motion is parallel to the principal motion axis. The second allowed motion direction is parallel to the image plane, because the underlying motion vector is designed to be perpendicular to the principal axis and the viewing direction.

\section{A. Motion-Model Generation}

The motion model is set up using a biplane 2-D/3-D registration of the previously generated 3-D catheter model to biplane fluoroscopic images acquired during a training phase. The images are processed as before, which leads to the distance transformed images for plane $\mathrm{A}, \mathbf{I}_{\mathrm{DT}, \mathrm{A}, t}$, and for plane $\mathrm{B}, \mathbf{I}_{\mathrm{DT}, \mathrm{B}, t}$, respectively, at time $t$. We allow for a full 3 -D search of the catheter model to get the best fit of the catheter model to each 2-D fluoroscopic image. In this case, the transformation matrix is written as

$$
\mathbf{T}_{u}(\mathbf{r})=\left(\begin{array}{cccc}
1 & 0 & 0 & r_{x} \\
0 & 1 & 0 & r_{y} \\
0 & 0 & 1 & r_{z} \\
0 & 0 & 0 & 1
\end{array}\right)
$$

with the translation parameters $\mathbf{r}=\left(r_{x}, r_{y}, r_{z}\right)^{T}$ and the index $u$ for 'unconstrained'. The cost function can be formulated as

$$
\begin{aligned}
& \hat{\mathbf{r}}=\arg \min _{\mathbf{r}} \sum_{i} \mathbf{I}_{\mathrm{DT}, \mathrm{A}, t}\left(\mathbf{P}_{A} \cdot \mathbf{T}_{u}(\mathbf{r}) \cdot \mathbf{m}_{i, t}\right) \\
&+ \mathbf{I}_{\mathrm{DT}, \mathrm{B}, t}\left(\mathbf{P}_{B} \cdot \mathbf{T}_{u}(\mathbf{r}) \cdot \mathbf{m}_{i, t}\right)
\end{aligned}
$$

with the projection matrices for image plane $\mathrm{A}, \mathbf{P}_{A}$, and plane $\mathrm{B}, \mathbf{P}_{B}$, as well as the 3 -D catheter model, $\mathbf{m}_{i, t}$, at time $t$. Optimization was performed using a multi-scale grid search approach [34]. The search space was sub-sampled and the region around the smallest cost function was used for a smaller search grid. The projection matrix used during the training phase tracking is not required to be one of the projection matrices used for model generation, i.e., the C-arm can be moved in between catheter model generation and the training phase. The same holds for the actual motion compensation. Given the parameters $\hat{\mathbf{r}}$ found by the nearest-neighbor search, the catheter model can be updated to $\mathbf{m}_{i, t+1} \in \mathbb{R}^{4}$ by

$$
\forall i: \mathbf{m}_{i, t+1}=\mathbf{T}_{u}(\hat{\mathbf{r}}) \cdot \mathbf{m}_{i, t} .
$$

During the training phase, the same transformation $\mathbf{T}_{u}(\hat{\mathbf{r}})$ can be applied to the 3-D volumetric data set that is used for image overlay. This way, a 3-D motion compensation can be shown during the training phase.

The patient specific motion model is calculated from the circumferential mapping catheter positions. To this end, the 


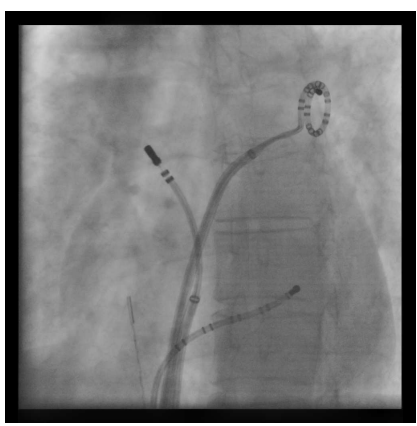

(a)

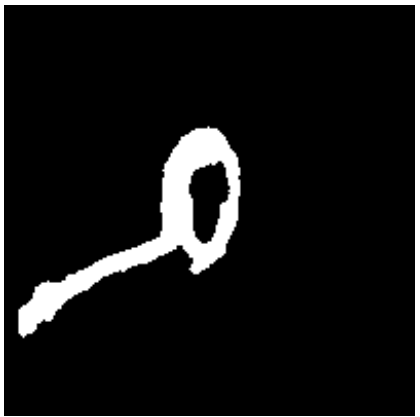

(d)

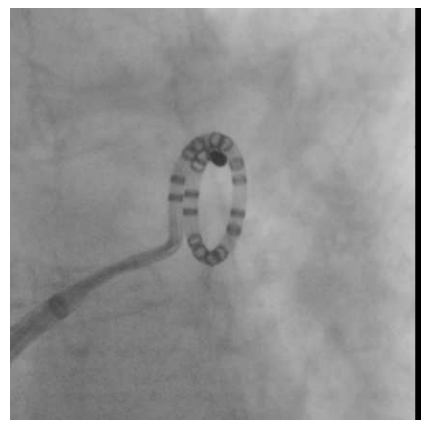

(b)

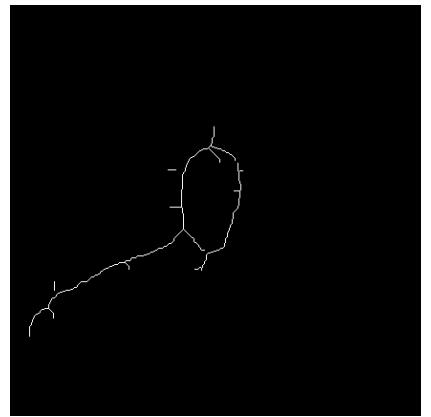

(e)

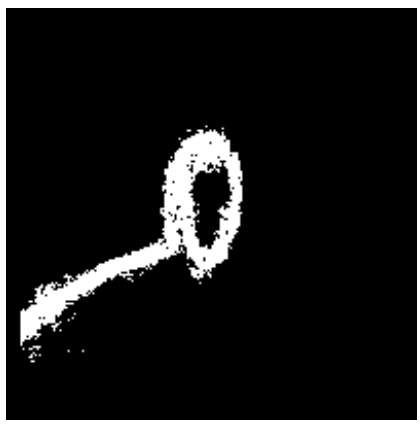

(c)

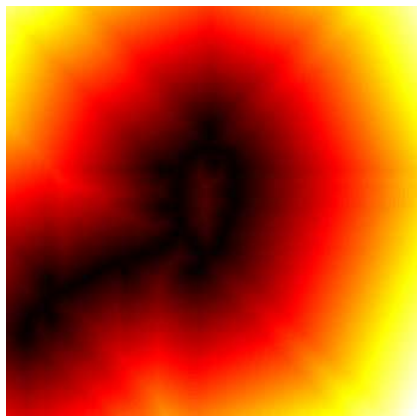

(f)

Fig. 3. Image processing on a fluoroscopic image. (a) The original fluoroscopic input image. (b) Cropped image around the region-of-interest. (c) Segmentation using a boosted classifier cascade. (d) Median filtered segmentation result. (e) Skeletonized image. (f) Distance transformed image $\mathbf{I}_{\mathrm{DT}}$.

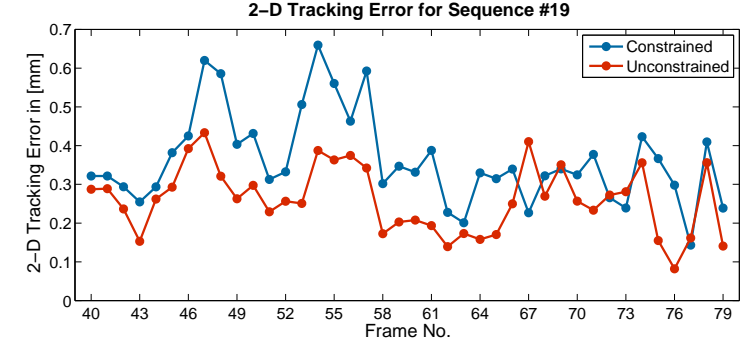

(a)

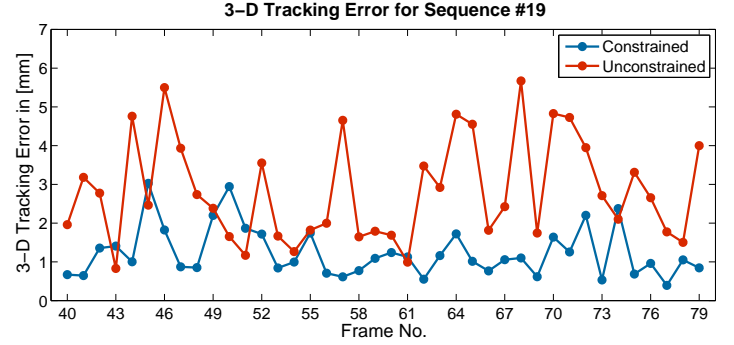

(b)

Fig. 4. (a) 2-D tracking error of the constrained and unconstrained approach for each frame of sequence \# 19. (b) 3-D tracking error of the constrained and unconstrained approach for the same sequence.

catheter model for every time step is reduced to the center of the model by

$$
\overline{\mathbf{m}}_{t}=\frac{1}{N} \sum_{i} \mathbf{m}_{i, t}
$$

The principal axis for the catheter centers $\overline{\mathbf{m}}_{t}$ is calculated by a principal component analysis, representing the main motion vector $\mathbf{v}_{m} \in \mathbb{R}^{3}$ with $\left\|\mathbf{v}_{m}\right\|_{2}=1$. For the motion model, only the principal axis is considered, as tracking inaccuracies during the training phase might produce outliers.

\section{B. Motion Compensation by Model-Constrained Registration}

In this section, motion compensation by model-constrained registration is introduced. The assumption for our approach is that only monoplane fluoroscopic imaging is available. Our proposed constraint is the reduction of the 3-D search space to a 2-D search space, by introducing a second feasible motion vector that is perpendicular to the viewing direction and the principal motion vector. This results in a 2-D search plane for the catheter model to be semi-parallel to the image plane. The cost function is the distance transform $\mathbf{I}_{\mathrm{DT}}$ of the post-processed segmentation result. By using the main motion vector, the 2-D search space also allows some depth estimation from a single X-ray view. A motion analysis of the left atrium, performed by Ector et al. [35], revealed that the dominant motion is in anterior-posterior and superior-inferior direction. They found that the degree of rotation is much less, and they attributed it to the deformation of the left atrium. Physicians position their $\mathrm{C}$-arms in standard viewing positions, usually only angulations in left-anterior-oblique (LAO), posterioranterior (PA), or right-anterior-oblique (RAO) direction are used. Angulations towards cranial or caudal directions are at least to the knowledge of the authors - not common for EP procedures. If image acquisition is performed with the Carm in an LAO, PA, or RAO position, most of the motion is captured, as the motion of the left atrium is parallel to the 


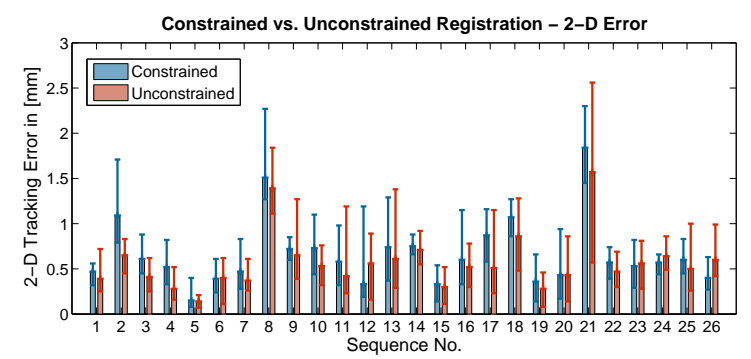

(a)

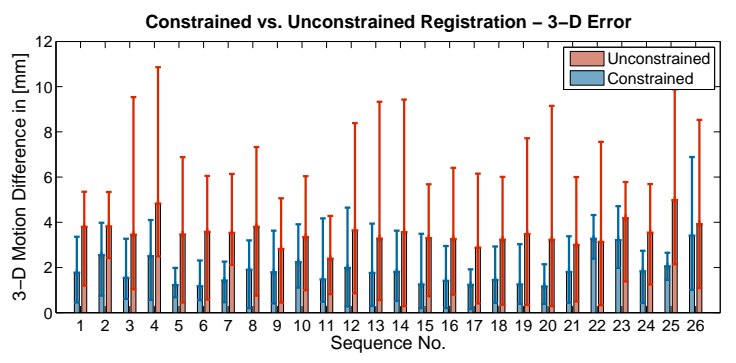

(c)

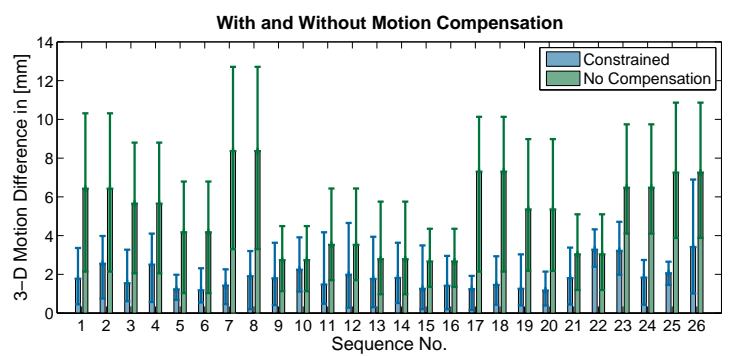

(b)

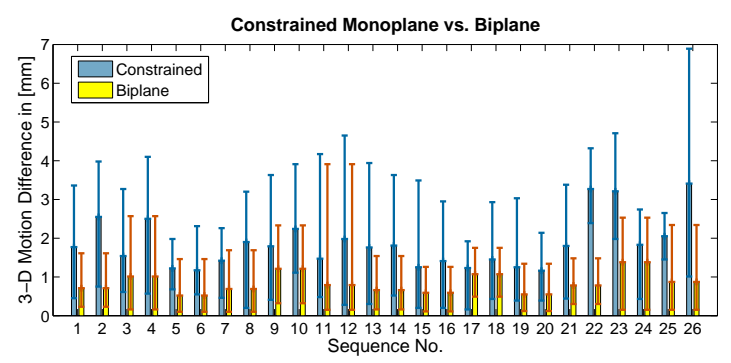

(d)

Fig. 5. (a) Comparison of the 2-D tracking accuracy of the constrained and unconstrained 2-D/3-D registration. (b) 3-D error for constrained motion compensation versus no motion compensation. (c) 3-D motion compensation error obtained for constrained 2-D/3-D registration in comparison to unconstrained 2-D/3-D registration. (d) Comparison of motion compensation between the constrained monoplane 2-D/3-D approach and the biplane approach.

image plane.

To carry out our constrained 2-D/3-D registration, we determine the viewing direction $\mathbf{v}_{v} \in \mathbb{R}^{3}$ with $\left\|\mathbf{v}_{v}\right\|_{2}=1$ of the optical axis from the last row of the projection matrix $\mathbf{P} \in \mathbb{R}^{3 \times 4}$ [36]. The second vector required to estimate the second search direction, which is perpendicular to the viewing direction and the main motion axis, is given by

$$
\mathbf{v}_{p}=\mathbf{v}_{m} \times \mathbf{v}_{v} .
$$

Any point on that plane can be represented by a linear combination of these two vectors $\mathbf{v}_{p}$ and $\mathbf{v}_{m}$. This translation can be rewritten in matrix notation as

$$
\mathbf{T}_{c}(\lambda, \mu)=\left(\begin{array}{cccc}
1 & 0 & 0 & \lambda v_{p, x}+\mu v_{m, x} \\
0 & 1 & 0 & \lambda v_{p, y}+\mu v_{m, y} \\
0 & 0 & 1 & \lambda v_{p, z}+\mu v_{m, z} \\
0 & 0 & 0 & 1
\end{array}\right)
$$

with $\mathbf{v}_{p}=\left(v_{p, x}, v_{p, y}, v_{p, z}\right)^{T}$ and $\mathbf{v}_{m}=\left(v_{m, x}, v_{m, y}, v_{m, z}\right)^{T}$ and the index $c$ for 'constrained'. The objective function for the constrained registration is then defined using the distance transformed image for image plane $\mathrm{A}, \mathbf{I}_{\mathrm{DT}, \mathrm{A}}$, or plane $\mathrm{B}$, $\mathbf{I}_{\mathrm{DT}, \mathrm{B}}$. In the remainder of this section, the indices $\mathrm{A}$ and $\mathrm{B}$ are omitted, and $\boldsymbol{P}$ stands either for $\boldsymbol{P}_{\mathrm{A}}$ or $\boldsymbol{P}_{\mathrm{B}}$. The same holds for $\boldsymbol{I}_{\mathrm{DT}, t}$. The cost function for the constrained registration can then be stated as

$$
\hat{\lambda}_{t}, \hat{\mu}_{t}=\arg \min _{\lambda, \mu} \sum_{i} \boldsymbol{I}_{\mathrm{DT}, t}\left(\boldsymbol{P} \cdot \boldsymbol{T}_{c}(\lambda, \mu) \cdot \mathbf{m}_{i, t}\right) .
$$

Optimization was performed using a nearest-neighbor search, as for the training phase [34]. Given the parameters $\hat{\lambda}_{t}, \hat{\mu}_{t}$, the catheter model can be updated to $\mathbf{m}_{i, t+1} \in \mathbb{R}^{4}$ by

$$
\forall i: \mathbf{m}_{i, t+1}=\mathbf{T}_{c}\left(\hat{\lambda}_{t}, \hat{\mu}_{t}\right) \cdot \mathbf{m}_{i, t} .
$$

The same transformation $\mathbf{T}_{c}\left(\hat{\lambda}_{t}, \hat{\mu}_{t}\right)$ is then applied to the 3-D volumetric data set that is used to compute the image overlay by 2-D forward projection of the 3-D model based on the known projection geometry. This way, we can achieve a 3-D motion compensation for monoplane fluoroscopic images.

\section{Motion Compensation by Unconstrained Registration}

The results of the constrained registration are compared to an unconstrained method that uses full 3-D translation as a motion model. To this end, an unconstrained registration to monoplane fluoroscopy is performed. In this case, Eq. 6 is adapted to the monoplane case by rewriting it as

$$
\hat{\mathbf{r}}^{\prime}=\arg \min _{\mathbf{r}} \sum_{i} \mathbf{I}_{\mathrm{DT}, t}\left(\mathbf{P} \cdot \mathbf{T}_{u}(\mathbf{r}) \cdot \mathbf{m}_{i, t}\right) .
$$

Motion compensation is then performed using $\hat{\mathbf{r}}^{\prime}$ to update the catheter model as in Eq. 7 and applying the same transformation to the 3-D data set used to generate the overlay images.

\section{Evaluation AND Results}

In this section, we evaluate the performance of our proposed motion-model constrained 2-D/3-D registration algorithm for motion compensation and present the results. The tracking accuracy of the constrained and unconstrained methods were calculated by comparison to a gold-standard segmentation. For evaluation, 13 clinical biplane sequences were available. The fluoroscopic sequences were acquired during standard electrophysiology procedures. The circumferential mapping catheter was placed at the ostium of the pulmonary vein during image acquisition. The catheter is usually firmly placed to ensure a good wall contact. A suboptimal wall contact may lead to undetected residual PV-atrial electrical connections, and potentially to an incomplete pulmonary vein isolation. One gold-standard segmentation was available for each sequence, i.e., the catheter was segmented by one expert observer in 
each frame of the whole sequence. Our data was taken from six different patients at one clinical site. All X-ray sequences were recorded on an AXIOM Artis dBC biplane C-arm system (Siemens AG, Healthcare Sector, Forchheim, Germany). The training of the classifier was performed on a two-fold cross validation, i.e., the biplane sequence considered for evaluation was excluded from the training data set. For each sequence, a 3-D model was generated as described in Sec. II Afterwards, the constrained method was evaluated by using each image plane of the biplane sequences independently. The frames used for the generation of the motion model were excluded from evaluation. For the unconstrained approach, the same frames were used for evaluation to arrive at comparable results. The constrained method used a training phase of $50 \%$ of the sequence. The shortest sequence available comprised 10 frames, and the longest 117. Individual sequences for training of the motion model were not available. To evaluate the influence of the number of frames used during the training phase, we took the three longest sequences available, consisting of 79, 95 and 117 frames, respectively. The training phases for this evaluation were chosen to comprise 5, 10, 20, 30, and 40 frames, respectively. The results are shown in Fig. 7 A 2-D tracking error was obtained by calculating the average 2-D distance of the projected catheter model to the gold-standard segmentation. The comparison of the 2-D tracking accuracy of both methods is shown in Fig. 5(a). The unconstrained method achieved an average 2-D tracking error of $0.57 \mathrm{~mm} \pm$ $0.31 \mathrm{~mm}$. The performance of the constrained method did not differ much and yielded a 2-D tracking error of $0.55 \mathrm{~mm} \pm$ $0.34 \mathrm{~mm}$. The frames of the training phase were not included.

Since the motion estimation and compensation is performed in 3-D, and for each case we have biplane sequences to derive the ground truth position in 3-D, a 3-D error can be estimated as well. To this end, the tip of the circumferential mapping catheter was manually localized in 3-D by triangulation from two views. This can only be used as an estimation for the actual 3-D error. An accurate evaluation would require a highresolution 3-D data set for each time instant. Such data is unfortunately not available. The 3-D trajectories of the catheter tip were taken as the gold-standard for the observed 3-D motion. For the 26 tested sequences, the observed motion was $4.5 \mathrm{~mm} \pm 2.4 \mathrm{~mm}$. The constrained motion compensation approach yielded a 3-D tracking error of $1.58 \mathrm{~mm} \pm$ $0.95 \mathrm{~mm}$. The unconstrained approach performed considerably worse with an average 3-D error of $3.21 \mathrm{~mm} \pm 1.62 \mathrm{~mm}$. Even though the constrained motion compensation method performed well, the gold-standard biplane method in [37] is still superior regarding the 3-D accuracy $(0.7 \mathrm{~mm} \pm 0.4 \mathrm{~mm})$. However, its better accuracy comes at the cost of increased $\mathrm{X}$-ray dose. A comparison of our constrained approach and the biplane approach is given in Fig. 5(d) In addition, a comparison of several motion compensation methods utilizing 2-D/3-D registration is given in Table III This includes the proposed constrained method, the unconstrained method, as well as the previously introduced reference biplane methods in [37] and [19].

As drift is an often discussed issue when evaluating tracking methods, we also considered the tracking error over time.

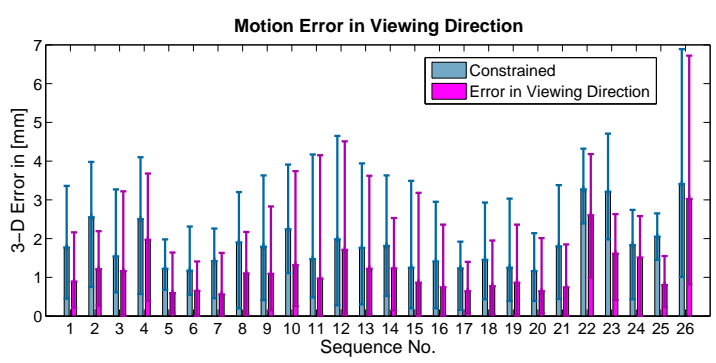

(a)

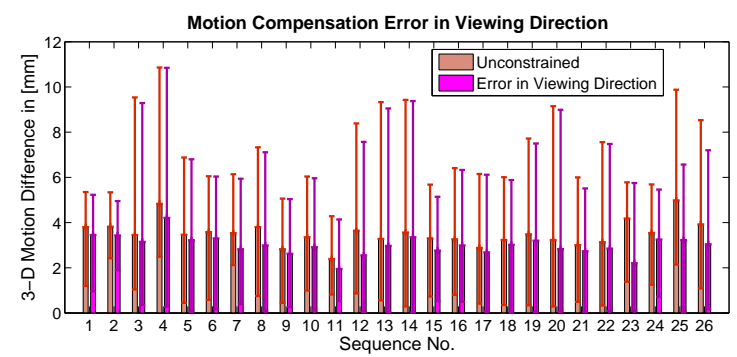

(b)

Fig. 6. Visualization of the motion compensation error in viewing direction. (a) The motion compensation error along the viewing direction of the constrained method. (b) The same graph for the unconstrained method.

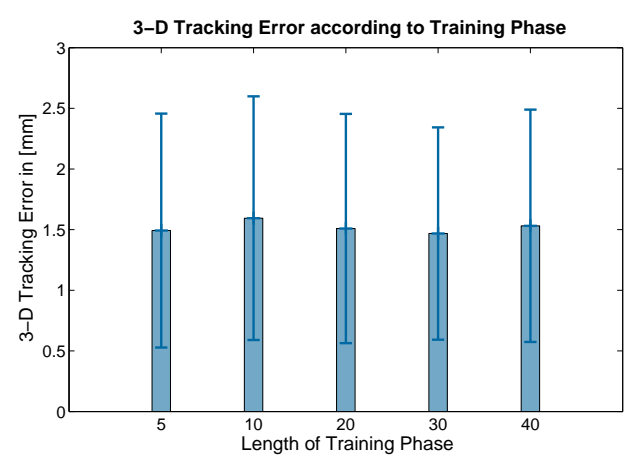

Fig. 7. Mean 3-D tracking error \pm standard deviation calculated over three sequences with 79,95 , and 117 frames versus different frame numbers used during the training phase.

This question is especially interesting, as only one previous frame is considered when tracking the current frame. In particular, the tracking result of the previous frame is used for cropping the region-of-interest in the current frame. Apart from that, all frames are treated independently. For example, the 2-D tracking error for sequence \# 19 is given in Fig. 4(a) Both the unconstrained and the constrained approach achieved comparable results with the constrained method yielding a slightly higher 2-D error. Specifically, in this particular sequence the 2-D tracking error was $0.36 \mathrm{~mm} \pm 0.12 \mathrm{~mm}$ for the constrained method and $0.26 \mathrm{~mm} \pm 0.09 \mathrm{~mm}$ for the unconstrained approach, respectively. The constrained method yielded a 3-D tracking error of $1.24 \mathrm{~mm} \pm 0.64 \mathrm{~mm}$, in comparison to the 3-D tracking error of $2.83 \mathrm{~mm} \pm 1.34 \mathrm{~mm}$ for the unconstrained method. Both methods did not suffer from drifting issues, suggesting that our model-based 2-D/3-D registration using a pre-generated 3-D catheter model is robust with respect to sporadic tracking errors. To further evaluate 


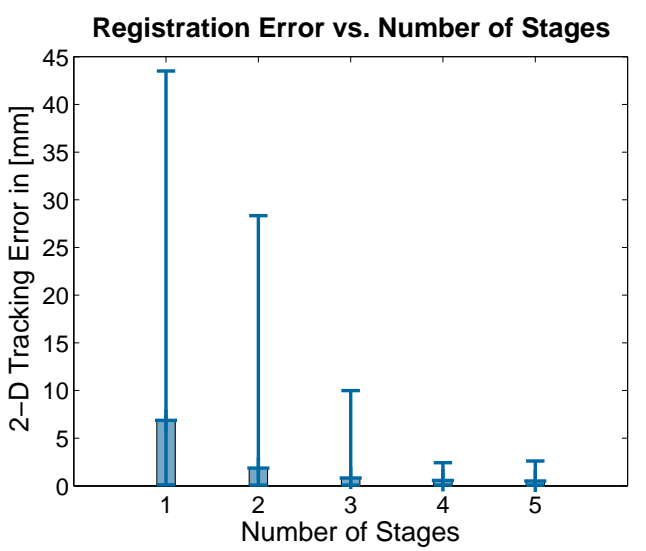

Fig. 9. 2-D tracking error versus the number of stages in the boosted classifier cascade.

how robust our method behaves against catheter model errors, the longest available sequence was chosen and the respective 3-D model was disturbed by Gaussian noise. Afterwards, tracking was performed and evaluated. The results are shown in Fig. 8

The error along the viewing direction was also computed. Since this direction is excluded in the search space for the constrained method, the error along the viewing direction was the largest among all directions, averaging at $1.03 \mathrm{~mm} \pm$ $0.94 \mathrm{~mm}$. However, the unconstrained method also had its largest error along the viewing direction with an average of $2.99 \mathrm{~mm} \pm 0.43 \mathrm{~mm}$, see Fig. 6(b), This confirms that estimating object depth from monoplane fluoroscopy is a challenging task as, e.g., pointed out in [38]. It also confirms that the constrained approach is a reasonable choice for tracking a mapping catheter put in place firmly at a pulmonary vein ostium.

The proposed method uses a boosted classifier cascade. To evaluate how many stages in the cascade are needed for achieving good motion compensation results, an experiment using the constrained method was performed. The results are shown in Fig. 9 With an increasing number of stages, the tracking accuracy improved. Using less than three stages yielded unsuccessful tracking results on our data set. According to our results and considering the fact that by using too many stages, we run the risk of overfitting the model to the noise in the training data, we propose to use 4 stages in the cascade. All results shown in this paper were obtained using 4 stages.

The number of stages used in the cascade could also be addressed by looking at the time required to estimate the motion for one frame. Fast and accurate methods are desired for interventional applications. We measured the time in $\mathrm{ms}$ for each frame and calculated the average, the minimum and the maximum. The results of the computational time versus the number of stages are given in Fig. 10. An example for segmentation results depending on the number of stages used for classification is shown in Fig. 11. Besides the runtime of the classification, the runtime of all components of our presented algorithm are also of interest. They are stated in Table \. Our time measurements were performed on an Intel

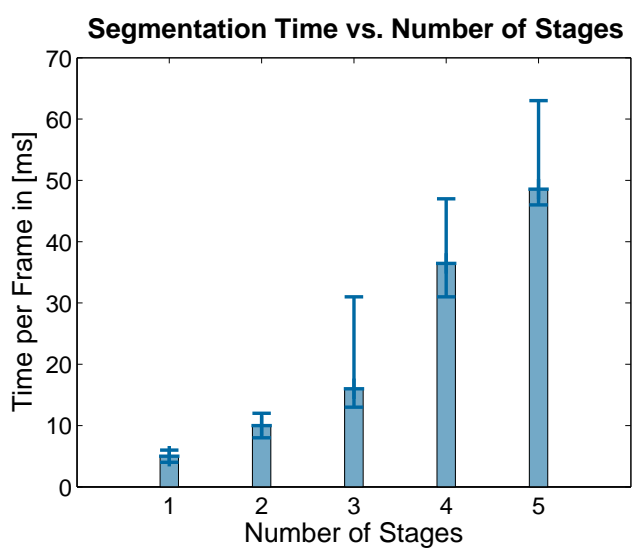

Fig. 10. Computational time of the catheter segmentation versus the number of stages in the boosted classifier cascade.

Xeon E5440 with $2.83 \mathrm{GHz}$.

\section{Discussion AND CONCLUSIONS}

The initialization of the catheter model used for motion compensation is required only once. The 2-D/3-D registration incorporates the projection matrix, so no model reinitialization is required if the viewing direction of a C-arm is changed. Although, model re-initialization is usually not time consuming, it does interrupt the workflow because manual interaction involving the user is required. In fact, while the catheter model can be calculated in less than $55 \mathrm{~ms}$ on our PC platform, user feedback needed for model initialization carries the risk that things are slowed down considerably. The accuracy of the model generation has already been evaluated in [19]. To further investigate the effect of catheter model errors, one sequence was tested with noisy input models. Gaussian noise with zero mean was used to disturb the model in 3-D. The results are shown in Fig. 8 It took a standard deviation of more than $3.0 \mathrm{~mm}$ to trigger tracking failures. And even then, they occurred in one image plane only demonstrating that a good view on an inaccurate catheter model may be able to work around this problem - only up to a certain degree of noise, of course. During our experiments, we found that a catheter model consisting of 50 points yielded good results. Increasing the number of model points further did not provide an increase tracking accuracy. Such a catheter model along with a disturbed catheter model is shown in Fig. 14

The 2-D tracking error of our proposed method is in the same range as that for the 2-D reference method [21], [27]. But instead of performing only a 2-D/2-D registration, we now rely on a constrained 2-D/3-D registration involving a 3-D catheter model as well as a motion model. The advantage of utilizing a 3-D catheter model is that catheter model re-initialization can be avoided when the $\mathrm{C}$-arm angulation changes during the intervention. Nevertheless, a sole 2-D approach may be the only option when only a monoplane fluoroscopic system is available, because the reconstruction of the 3-D catheter model requires at least two views in the same cardiac cycle and breathing phase. The computation of the motion model 


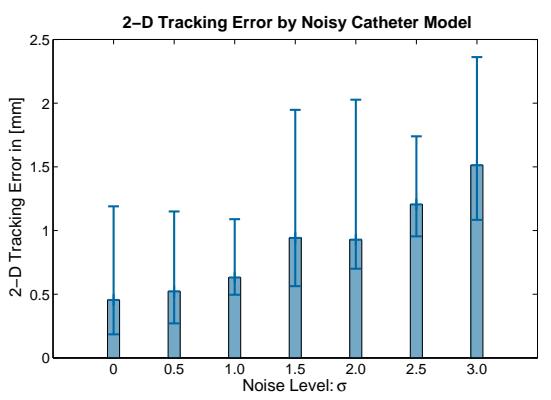

(a)

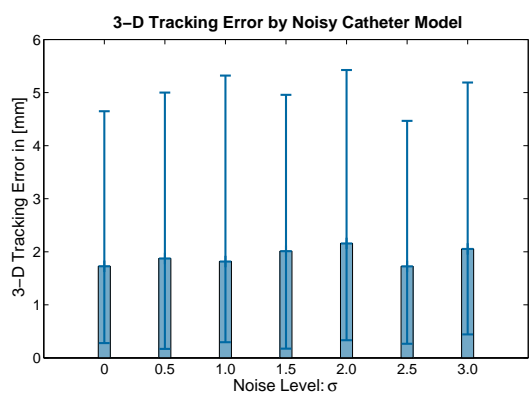

(b)

Fig. 8. Tracking error with respect to a noise catheter model. (a) 2-D tracking error. (b) 3-D tracking error.

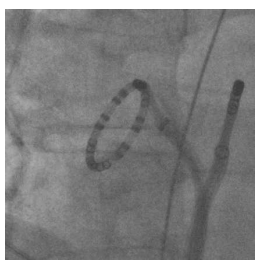

(a)

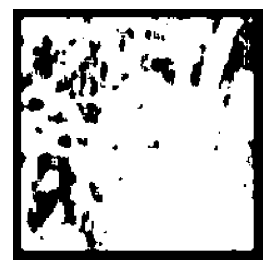

(b)

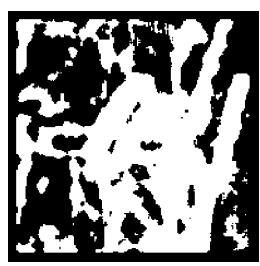

(c)

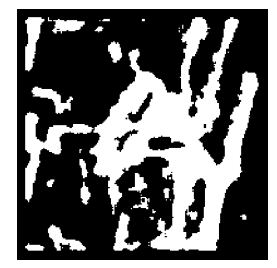

(d)

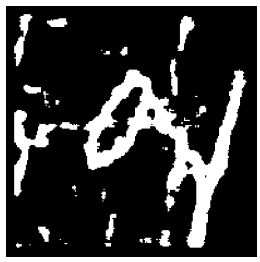

(e)

Fig. 11. Segmentation results for different number of cascades. The segmentation results shown here are smoothed by a median filter. (a) Cropped image used for segmentation. (b) Segmentation result with one stage in the cascade. (c) Segmentation with two stages. (d) Segmentation with three stages. (e) Segmentation with four stages.

TABLE I

RUNTIME OF ALGORITHM COMPONENTS

\begin{tabular}{|l|c|}
\hline \multicolumn{2}{|c|}{ Runtime } \\
\hline \hline Components & Runtime in [ms] \\
\hline Segmentation & $\sim 42 \mathrm{~ms}$ \\
\hline Median & $<1 \mathrm{~ms}$ \\
\hline Skeletonization & $\sim 47 \mathrm{~ms}$ \\
\hline Distance Transform & $<1 \mathrm{~ms}$ \\
\hline Constrained Registration & $\sim 17 \mathrm{~ms}$ \\
\hline
\end{tabular}

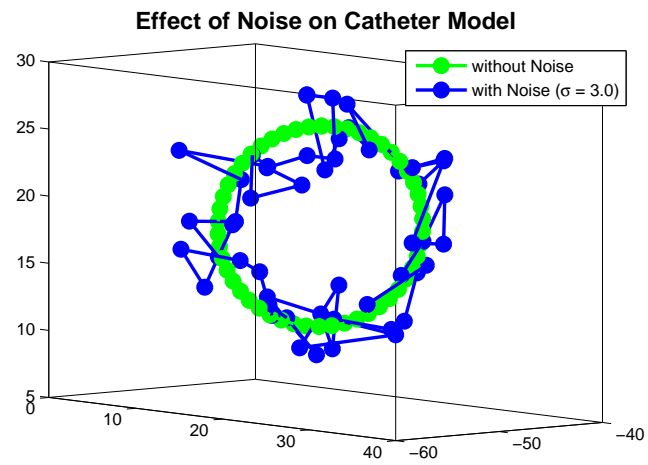

Fig. 14. Comparison between a catheter model with and without noise. The noisy catheter model was disturbed by Gaussian noise with zero mean and a standard deviation of $3.0 \mathrm{~mm}$.

requires a training phase. We used $50 \%$ of the available biplane sequence to compute the principal motion axis. In clinical practice, this could be included into the workflow. At the beginning of each AFib ablation procedure, the signals at the PVs are documented and the correct position of the circumferential mapping catheter is verified by contrast injection and
TABLE II

COMPARISON BETWEEN SEVERAL METHODS ON MOTION COMPENSATION USING 2-D/3-D REGISTRATION

\begin{tabular}{|c|c|c|c|c|}
\hline \multicolumn{5}{|c|}{ Method Comparison } \\
\hline \hline & Constrained & Unconstrained & {$[37$} & {$[\mathbf{1 9}]$} \\
\hline & Monoplane & Monoplane & Biplane & Biplane \\
\hline 2-D Error: & $0.6 \mathrm{~mm}$ & $0.6 \mathrm{~mm}$ & $0.8 \mathrm{~mm}$ & $1.0 \mathrm{~mm}$ \\
\hline 3-D Error: & $1.6 \mathrm{~mm}$ & $3.2 \mathrm{~mm}$ & $0.7 \mathrm{~mm}$ & $0.8 \mathrm{~mm}$ \\
\hline
\end{tabular}

- if available - using a short biplane sequence. This sequence might already be sufficient to set up our proposed motion model. As four pulmonary veins are to be ablated during the procedure, it might be necessary to train four individual motion models, i.e., one for each of the PVs. Evaluating the 3-D tracking error with respect to number of frames used during the training phase, we conclude that our method is insensitive to the length of the training phase, as shown in Fig. 7. Even though a short sequence might be sufficient to estimate the principle direction of the motion, a full breathing cycle should be used for best results. For example, if the patient is consciously sedated, the physician could ask the patient perform a deep inhale and exhale during the training sequence for the motion model. Using general anesthesia, this might not be required.

Our proposed method is able to achieve a 3-D accuracy of about $1.6 \mathrm{~mm}$. Unfortunately, there is hardly a statement by a physician about the amount of error that is clinically acceptable. For cardiac applications though, $2 \mathrm{~mm}$ seems to be an accepted threshold [39]. Nevertheless, to reduce the 3-D error, one could employ simultaneous biplane imaging which comes at the cost of a higher dose for patient and the medical staff [37]. As physicians are used to 2-D projection images and the 2-D error is lower, it is an open question whether a 


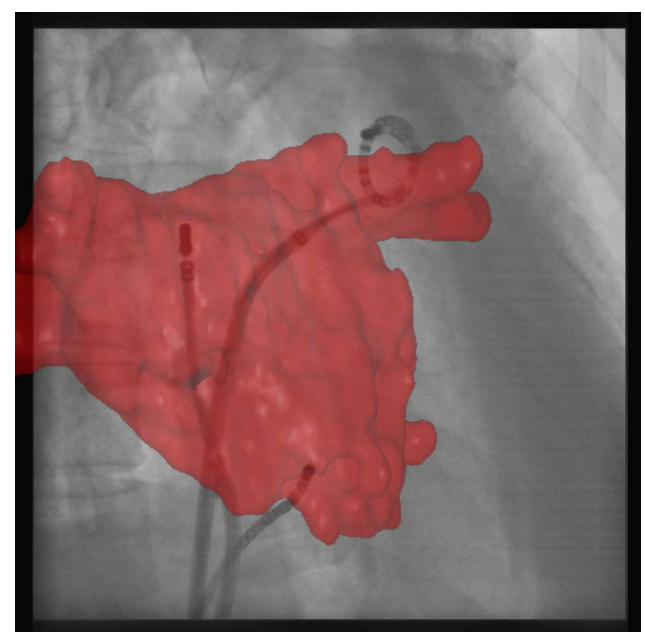

(a)

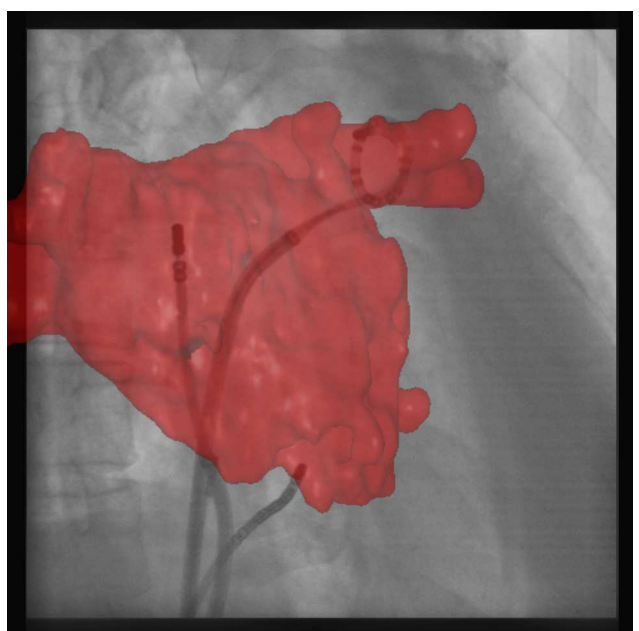

(b)

Fig. 12. A comparison showing the difference whether or not motion compensation is applied on the fluoroscopic overlay. (a) One frame of sequence 17 without motion compensation. (b) The same frame of sequence 17 with motion compensation.

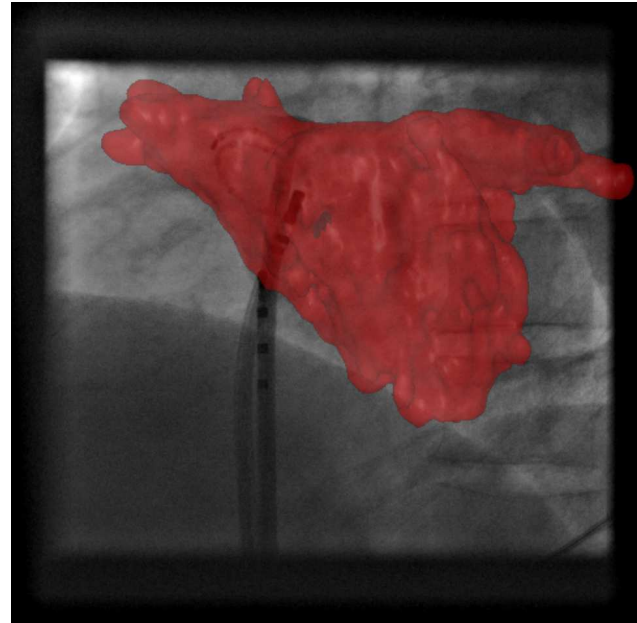

(a)

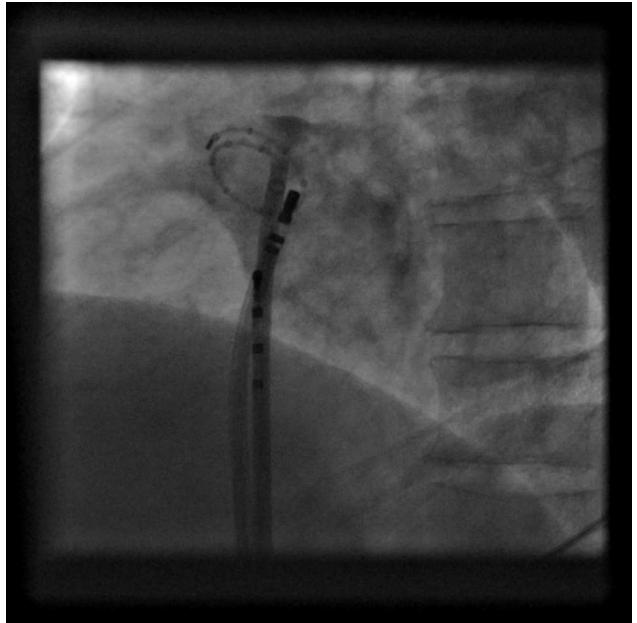

(b)

Fig. 13. Visual inspection of the motion compensation method. (a) One motion compensated frame of one sequence with 3-D overlay during contrast injection close to one pulmonary vein. (b) The same frame without the 3-D overlay.

3-D error of $2 \mathrm{~mm}$ can be accepted or not. It seems as if a clinical evaluation of the proposed method would have to be performed in order to evaluate the clinically required accuracy.

The limitation of our method is mainly related to the fact that motion along the viewing direction cannot be taken into account because it is difficult to estimate depth information reliably from monoplane projection images [38], [40]. Using an unconstrained approach, the 3-D error remains high, especially along the viewing direction, see Fig. 6. Depth correction could be performed by analyzing the width of the object. But this requires a perfect segmentation of the catheter from the fluoroscopy views, which is difficult for low-dose $\mathrm{X}$-ray images. In addition, we would also need to know the exact dimensions of the catheter in 3-D, i.e., its diameter and thickness. Any noise or inaccuracy in the 2-D segmentation or the 3-D model would significantly deteriorate the accuracy of depth estimation. Even if the depth information could be accurately estimated, the effect would probably not be very visible because the size of the overlay would only change slightly. Nevertheless, 3-D motion errors in X-ray viewing direction are a major contributing factor why the unconstrained method yields significantly worse results, see Fig. 5(c), Our proposed method does not need an explicit depth-estimation step thanks to the motion-model. If there is a significant motion in X-ray view direction, then it will be captured by the main motion axis. The distance transform provides the main input for the cost function. As long as only one circumferential mapping catheter appears in the image, there is only one global optimum for the cost function. Using our multi-scale grid-search approach, we did not run into local optima. Some of these occur around the region of the correct position. If multiple elliptical shaped catheters were used, more local optima would appear and our optimization strategy could run into one of these. This restricts our method to cases using a single circumferential mapping catheter. Fortunately, the majority of AFib cases belong to this category. A visualization 


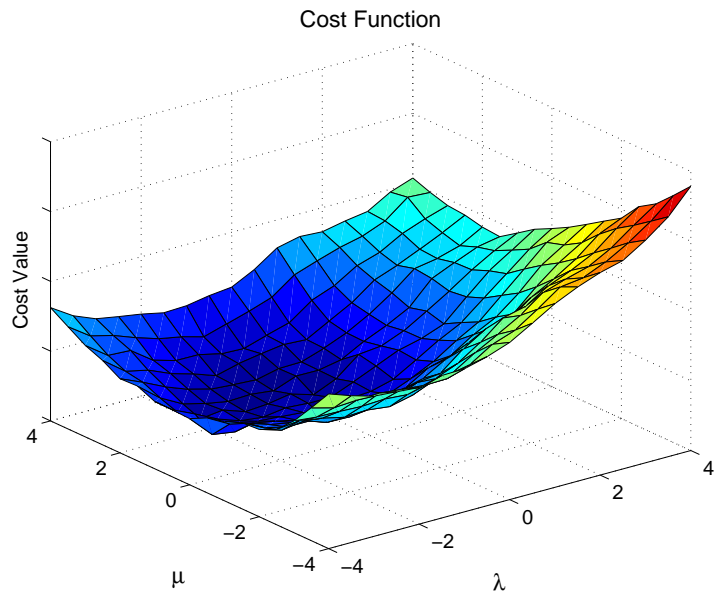

Fig. 15. The values of the cost function for one frame in a small area around the optimum. The global optimum is at $\mu=1.0 \mathrm{~mm}$ and $\lambda=-0.5 \mathrm{~mm}$.

of the cost function is given in Fig. 15

Other 2-D/3-D registration approaches [41], [42] have not been tried yet. Since we are dealing with a very small structure, they are difficult to apply. Although the method in [43], [44] is similar to our approach in spirit, it involves a direct imageto-image similarity measure which we find more difficult to evaluate than our current approach.

One gold standard database comprising 938 frames was available for training. The training on a larger database would further improve the segmentation results. The more training samples we have, the more likely we are to capture most of the subtle differences. This is particularly important in difficult cases where contrast may be low. This can happen when treating heavy patients, e.g., due to scatter radiation. [45]. Our data set comprised biplane fluoroscopic images of six patients. We encountered two different types of circumferential mapping catheters. One type was used in 11 biplane sequences, and a second type was used in two more sequences.

Other methods for image-based respiratory motion compensation in electro-physiology procedures have been proposed as well [46], [47]. The first method uses a different catheter and the second involves a pre-operative data set. The main shortcoming of these methods is that they do not estimate the motion at the site of ablation directly. Therefore, they require either a patient-specific model built beforehand, or a heuristic prior to infer the motion at the site of ablation from the motion estimates. Since the motion estimates appear to be joint estimates of heart and breathing motion, the two motion components need to be separated for respiratory motion correction. When the motion is estimated in $2-\mathrm{D}$, reinitialization is required whenever when the $\mathrm{C}$-arm position changes. Our proposed method, on the other hand, is captures the relevant motion right at the site of ablation and takes it into account real-time. Since our approach uses a 3-D catheter model, re-initialization after repositioning the C-arm can be avoided. For comparison, non-image-based methods for motion compensation involving electro-anatomic mapping systems provide a 3-D mean tracking error of $0.7 \mathrm{~mm}$ [48] which is comparable to our mean 2-D tracking error of
$0.55 \mathrm{~mm}$. Since we do not need to record the ECG signal, a stand-alone version of our motion-compensated fluoroscopy system is more straightforward. A comparison of different methods to perform motion compensation is given in [49].

Our method is purely image driven. Considering the catheters available during AFib ablation procedures, the only other possible catheter candidate to perform motion compensation with is the catheter in the coronary sinus (CS), as proposed in [46]. Our proposed method could be extended to learn the motion difference between the circumferential mapping and the CS catheter. The same idea could be applied to using the diaphragm for motion compensation. Our current implementation for motion estimation relies on the assumption that the circumferential mapping catheter is firmly placed at the PV where ablation takes place. If the mapping catheter floated around freely within the left atrium, we would not get a reliable motion estimate with our current method. In such a case, we would need to introduce an additional motion analysis stage to detect the free motion.

Apart from a filter-based approach, no other learning-based methods have been tried yet. It has been shown that learningbased methods can be superior to filter-based methods [37]. Here, the filter-based approach [19] yielded a 2-D tracking error of $1.0 \mathrm{~mm}$ and a 3-D tracking error of $0.8 \mathrm{~mm}$, respectively. Using a learning-based method, the errors were reduced to $0.8 \mathrm{~mm}$ in 2-D and to $0.7 \mathrm{~mm}$ in 3-D [37]. To further improve the accuracy, other methods such as probabilistic boosting trees [50] or random forests [51] for catheter segmentation could be considered. For a higher efficiency, a different skeletonization method other than the thinning algorithm in [32] should be considered, as this method is currently the bottleneck of our approach regarding computational efficiency. Since the goal of motion compensation for AFib ablation procedures is to work within an interventional setup, near real-time performance is desirable. In this context, real-time is regarded as the frame rate that is used for image acquisition during the intervention. AFib ablation procedures are lengthy procedures, and fluoroscopy times often accumulate to more than 30 minutes. This is why physicians try to reduce dose, e.g., by lowering the acquisition frame rates. For example, some centers use frame rates as low as 1 frame per second (fps). A more typical frame rate is $3 \mathrm{fps}$. Frame rates exceeding 15 fps are highly unusual. One reason for the long fluoroscopy times is the complexity encountered when trying to isolate the pulmonary veins. We hope to shorten the procedure time by offering better navigation based on fluoroscopy overlay images, but further clinical studies are needed to confirm this. As of now, there are some some published results indicating that X-ray based navigation can at least shorten procedure time, e.g., when compared to the CARTO electromagnetic tracking system [52].

A comparison between an overlay with and without motion compensation is presented in Fig. 12, In Fig. 13, a fluoroscopic image with a motion-compensated 3-D overlay is compared to the original X-ray frame using a contrast injection. In a clinical setup, a physician working on a biplane system is likely to use the two X-ray image planes in an alternating way. For such a clinical use case, our newly proposed method 
provides a significant advantage over the previously introduced methods [18], [37], [19], [27], [21] in terms of accuracy and practicality. Furthermore, a combination of the proposed constrained method and the previous biplane reference approach in [37] might provide a seamless workflow and high degree of flexibility to the physicians. For example, during regular procedures, the constrained method could be used. If a higher accuracy is required, physicians can switch to a biplane fluoroscopy and the method in [37] may start automatically from the initial position provided by the constrained method.

\section{ACKNOWLEDGEMENTS}

The authors gratefully acknowledge funding of the Erlangen Graduate School in Advanced Optical Technologies (SAOT) by the German Research Foundation (DFG) in the framework of the German excellence initiative. This work has been supported by the German Federal Ministry of Education and Research (BMBF), project grant No. 01EX1012E, in the context of the initiative Spitzencluster Medical Valley - Europäische Metropolregion Nürnberg. Additional funding was provided by Siemens AG, Healthcare Sector.

\section{REFERENCES}

[1] B. Gage, A. Waterman, W. Shannon, M. Boechler, M. Rich, and M. Radford, "Validation of Clinical Classification Schemes for Predicting Stroke," Journal of the American Medical Association, vol. 285, no. 22, pp. 2864-2870, June 2001.

[2] M. Haissaguerre, L. Gencel, B. Fischer, P. L. Metayer, F. Poquet, F. I. Marcus, and J. Clementy, "Successful catheter ablation of atrial fibrillation," Journal of Cardiovascular Electrophysiology, vol. 5, no. 12, pp. 1045-1052, December 1994.

[3] H. Calkins, J. Brugada, D. Packer, R. Cappato, S. Chen, H. Crijns, R. Damiano, D. Davies, D. Haines, M. Haissaguerre, Y. Iesaka, W. Jackman, P. Jais, H. Kottkamp, K. Kuck, B. Lindsay, F. Marchlinski, P. McCarthy, J. Mont, F. Moradi, K. Nademanee, A. Natale, C. Pappone, E. Prystowsky, A. Raviele, J. Ruskin, and R. Shemin, "HRS/EHRA/ECAS Expert Consensus Statement on Catheter and Surgical Ablation of Atrial Fibrillation: Recommendations for Personnel, Policy, Procedures and Follow-Up," Europace, vol. 9, no. 6, pp. 335379, June 2007.

[4] O. Wazni, N. Marrouche, D. Martin, A. Verma, M. Bhargava, W. Saliba, D. Bash, R. Schweikert, J. Brachmann, J. Gunther, K. Gutleben, E. Pisano, D. Potenza, R. Fanelli, A. Raviele, S. Themistoclakis, A. Rossillo, A. Bonso, and A. Natale, "Radiofrequency ablation vs antiarrhythmic drugs as first-line treatment of symptomatic atrial fibrillation: a randomized trial," Journal of the American Medical Association, vol. 293, no. 21, pp. 2634-2640, January 2005.

[5] R. Cappato, H. Calkins, S.-A. Chen, W. Davies, Y. Iesaka, J. Kalman, Y.-H. Kim, G. Klein, D. Packer, and A. Skanes, "Worldwide Survey on the Methods, Efficacy, and Safety of Catheter Ablation for Human Atrial Fibrillation," Circulation, vol. 111, pp. 1100-1105, February 2005.

[6] M. Prümmer, L. Wigström, J. Hornegger, J. Boese, G. Lauritsch, N. Strobel, and R. Fahrig, "Cardiac C-arm CT: Efficient Motion Correction for 4D-FBP," in IEEE Nuclear Science Symposium Conference Record, Oct. 29 - Nov. 12006.

[7] M. Prümmer, R. Fahrig, L. Wigström, J. Boese, G. Lauritsch, N. Strobel, and J. Hornegger, "Cardiac c-arm ct: 4d non-model based heart motion estimation and its application," in Proc. of SPIE Medical Imaging 2007: Physics of Medical Imaging, ser. 651015, J. Hsieh and M. Flynn, Eds., vol. 6510, February 2007, p. 651015 .

[8] M. Prümmer, J. Hornegger, G. Lauritsch, L. Wigström, E. GirardHughes, and R. Fahrig, "Cardiac C-arm CT: a unified framework for motion estimation and dynamic CT," IEEE Transactions on Medical Imaging, vol. 28, no. 11, pp. 1836-1849, November 2009.
[9] N. Strobel, O. Meissner, J. Boese, T. Brunner, B. Heigl, M. Hoheisel, G. Lauritsch, M. Nagel, M. Pfister, E.-P. Rührnschopf, B. Scholz, B. Schreiber, M. Spahn, M. Zellerhoff, and K. Klingenbeck-Regn, "Imaging with Flat-Detector C-Arm Systems," in Multislice CT (Medical Radiology / Diagnostic Imaging), 3rd ed., M. F. Reiser, C. R. Becker, K. Nikolaou, and G. Glazer, Eds. Springer Berlin / Heidelberg, January 2009, ch. 3, pp. 33-51.

[10] F. Wittkampf, E. Wever, R. Derksen, A. Wilde, H. Ramanna, R. Hauer, and E. Robles de Medina, "LocaLisa - New Technique for RealTime 3-Dimensional Localization of Regular Intracardiac Electrodes," Circulation, vol. 99, no. 13, pp. 1312-1317, 1999.

[11] P. Kistler, K. Rajappan, M. Jahngir, M. Earley, S. Harris, D. Abrams, D. Gupta, R. Liew, S. Ellis, S. Sporton, and R. Schilling, "The Impact of CT Image Integration into an Electroanatomic Mapping System on Clinical Outcomes of Catheter Ablation of Atrial Fibrillation," Journal of Cardiovascular Electrophysiology, vol. 17, no. 10, pp. 1093-1101, October 2006

[12] P. Kistler, M. Earley, S. Harris, D. Abrams, S. Ellis, S. Sporton, and R. Schilling, "Validation of Three-Dimensional Cardiac Image Integration: Use of Integrated CT Image into Electroanatomic Mapping System to Perform Catheter Ablation of Atrial Fibrillation," Journal of Cardiovascular Electrophysiology, vol. 17, no. 4, pp. 341-348, April 2006.

[13] P. Kistler, K. Rajappan, S. Harris, M. Earley, L. Richmond, S. Sporton, and R. Schilling, "The impact of image integration on catheter ablation of atrial fibrillation using electroanatomic mapping: a prospective randomized study," European Heart Journal, vol. 29, no. 24, pp. 30293036, October 2008.

[14] M. Daccarett, N. Segerson, J. Günther, G. Nölker, K. Gutleben, J. Brachmann, and N. Marrouche, "Blinded correlation study of threedimensional electro-anatomical image integration and phased array intracardiac echocardiography for left atrial mapping," Europace, vol. 9, pp. 923-926, September 2007.

[15] J. Ector, S. De Buck, W. Huybrechts, D. Nuyens, S. Dymarkowski, J. Bogaert, F. Maes, and H. Heidbüchel, "Biplane three-dimensional augmented fluoroscopy as single navigation tool for ablation of atrial fibrillation: Accuracy and clinical value," Heart Rhythm, vol. 5, no. 7, pp. 957-964, March 2008.

[16] J. Sra, G. Narayan, D. Krum, A. Malloy, R. Cooley, A. Bhatia, A. Dhala, Z. Blanck, V. Nangia, and M. Akhtar, "Computed TomographyFluoroscopy Image Integration-Guided Catheter Ablation of Atrial Fibrillation," Journal of Cardiovascular Electrophysiology, vol. 18, no. 4, pp. 409-414, April 2007.

[17] S. De Buck, F. Maes, J. Ector, J. Bogaert, S. Dymarkowski, H. Heidbüchel, and P. Suetens, "An Augmented Reality System for Patient-Specific Guidance of Cardiac Catheter Ablation Procedures," IEEE Transactions on Medical Imaging, vol. 24, no. 11, pp. 1512-1524, November 2005.

[18] A. Brost, R. Liao, J. Hornegger, and N. Strobel, "3-D Respiratory Motion Compensation during EP Procedures by Image-Based 3-D Lasso Catheter Model Generation and Tracking," in 12th International Conference on Medical Image Computing and Computer-Assisted Intervention (MICCAI) 2009, London, UK, ser. Lecture Notes in Computer Science, G.-Z. Yang, D. Hawkes, D. Rueckert, J. Noble, and C. Taylor, Eds. Springer Berlin / Heidelberg, 2009, vol. 5761, pp. 394-401.

[19] A. Brost, R. Liao, N. Strobel, and J. Hornegger, "Respiratory motion compensation by model-based catheter tracking during EP procedures," Medical Image Analysis, vol. 14, no. 5, pp. 695-706, 2010, special Issue on the 12th International Conference on Medical Image Computing and Computer-Assisted Intervention (MICCAI) 2009.

[20] M. Truong, A. Aslam, M. Ginks, C. Rinaldi, R. Rezavi, G. Penney, and K. Rhode, "2D-3D registration of cardiac images using catheter constraints," in Computers in Cardiology, September 2009, pp. 605 608.

[21] A. Brost, R. Liao, J. Hornegger, and N. Strobel, "Model-Based Registration for Motion Compensation during EP Ablation Procedures," in Biomedical Image Registration, ser. Lecture Notes in Computer Science, B. Fischer, B. Dawant, and C. Lorenz, Eds. Springer Berlin / Heidelberg, 2010, vol. 6204, pp. 234-245.

[22] G. Shechter, B. Shechter, J. R. Resar, and R. Beyar, "Prospective motion correction of x-ray images for coronary interventions," IEEE Transactions on Medical Imaging, vol. 24, no. 4, pp. 441-450, April 2005.

[23] R. Halir and J. Flusser, "Numerically Stable Direct Least Squares Fitting Of Ellipses," in In Proceedings of the 6th Conference in Central Europe on Computer Graphics and Visualization, Plzen, February 1998, pp. 253-257. 
[24] D.Zwillinger, CRC Standard Mathematical Tables and Formulae, 31st ed. CRC Press Boca Raton, 2002.

[25] L. Quan, "Conic Reconstruction and Correspondence From Two Views," IEEE Transactions on Pattern Analysis and Machine Intelligence, vol. 18, no. 2, pp. 151-160, February 1996.

[26] A. Brost, N. Strobel, L. Yatziv, W. Gilson, B. Meyer, J. Hornegger, J. Lewin, and F. Wacker, "Geometric Accuracy of 3-D X-Ray ImageBased Localization from Two C-Arm Views," in Workshop on Geometric Accuracy In Image Guided Interventions - Medical Image Computing and Computer Assisted Interventions 2009. London UK: MICCAI, September 2009, pp. 12-19.

[27] A. Brost, A. Wimmer, R. Liao, J. Hornegger, and N. Strobel, "Motion compensation by registration-based catheter tracking," K. Wong and D. Holmes, Eds., vol. 7964, no. 1. SPIE, 2011, p. 79641 O.

[28] Y. Freund and R. Schapire, "A decision-theoretic generalization of online learning and an application to boosting," Journal of Computer and System Sciences, vol. 55, no. 1, pp. 119-139, August 1997.

[29] J. Friedman, T. Hastie, and R. Tibshirani, "Additive Logistic Regression: a Statistical View of Boosting," Annals of Statistics, vol. 28, no. 2, pp. $337-407,2000$.

[30] P. Viola and M. Jones, "Robust real-time face detection," International Journal of Computer Vision, vol. 57, no. 2, pp. 137-154, 2004.

[31] L. Breiman, J. Friedman, R. Olshen, and C. Stone, Classification and Regression Trees. New York, USA: Chapman \& Hall, 1984.

[32] J. M. Cychosz, Efficient Binary Image Thinning using Neighborhood Maps. San Diego, CA, USA: Academic Press Professional, Inc., 1994, pp. $465-473$.

[33] H. Breu, J. Gil, D. Kirkpatrick, and M. Werman, "Linear time Euclidean distance transform algorithms," IEEE Transactions on Pattern Analysis and Machine Intelligence, vol. 17, no. 5, pp. 529-533, May 1995.

[34] R. Duda, P. Hart, and D. Stork, Pattern Classification, 2nd ed. John Wiley \& Sons, Inc, August 2000.

[35] J. Ector, S. De Buck, D. Loeckx, W. Coudyzer, F. Maes, S. Dymarkowski, J. Bogaert, and H. Heidbüchel, "Changes in left atrial anatomy due to respiration: Impact on three-dimensional image integration during atrial fibrillation ablation," Journal of Cardiovascular Electrophysiology, vol. 19, no. 8, pp. 828-834, August 2008.

[36] R. Hartley and A. Zisserman, Multiple View Geometry in Computer Vision, 2nd ed. Cambridge University Press, Cambridge, March 2004.

[37] A. Brost, A. Wimmer, R. Liao, J. Hornegger, and N. Strobel, "Catheter Tracking: Filter-Based vs. Learning-Based," in Pattern Recognition, ser. Lecture Notes in Computer Science, M. Goesele, S. Roth, A. Kuijper, B. Schiele, and K. Schindler, Eds. Springer Berlin / Heidelberg, 2010, vol. 6376, pp. 293-302.

[38] P. Fallavollita, "2D/3D registration using only single-view fluoroscopy to guide cardiac ablation procedures: a feasibility study," in Medical Imaging 2010: Visualization, Image-Guided Procedures, and Modeling, vol. 7625 , no. 1. SPIE, February 2010, p. 762507.

[39] M. Esteghamatian, Z. Azimifar, P. Radau, and G. Wright, "Real-time $2 \mathrm{~d}-3 \mathrm{~d} \mathrm{mr}$ cardiac image registration during respiration using extended kalman filter predictors," in 9th International Conference on Signal Processing (ICSP 2008). Bejing, China: IEEE, October 26 - 29 2008, pp. $1325-1328$.

[40] P. Fallavollita, "Is single-view fluoroscopy sufficient in guiding cardiac ablation procedures?" Journal of Biomedical Imaging, pp. 1:1 - 1:13, March 2010, Article ID 631264.

[41] G.-A. Turgeon, G. Lehmann, G. Guiraudon, M. Drangova, D. Holdsworth, and T. Peters, " $2 \mathrm{~d}-3 \mathrm{~d}$ registration of coronary angiograms for cardiac procedure planning and guidance," Medical Physics, vol. 32, no. 12, pp. 3737-3749, December 2005.

[42] J. Yao and R. Taylor, "Assessing accuracy factors in deformable $2 \mathrm{~d} / 3 \mathrm{~d}$ medical image registration using a statistical pelvis model," in Proceedings of Ninth IEEE International Conference on Computer Vision, vol. 2, October 2003, pp. $1329-1334$.

[43] J. Hermans, P. Claes, J. Bellemans, D. Vandermeulen, and P. Suetens, "A robust optimization strategy for intensity-based $2 \mathrm{~d} / 3 \mathrm{~d}$ registration of knee implant models to single-plane fluoroscopy," Proc. SPIE Medical Imaging 2007, vol. 6512, no. 1, p. 651227, 2007.

[44] M. Mahfouz, W. Hoff, R. Komistek, and D. Dennis, "A robust method for registration of three-dimensional knee implant models to twodimensional fluoroscopy images," IEEE Transactions on Medical Imaging, vol. 22, no. 12, pp. 1561 - 1574, December 2003.

[45] N. Strobel, O. Meissner, J. Boese, T. Brunner, B. Heigl, M. Hoheisel, G. Lauritsch, M. Nagel, M. Pfister, E.-P. Rührnschopf, B. Scholz, B. Schreiber, M. Spahn, M. Zellerhoff, and K. Klingenbeck-Regn, "Imaging with Flat-Detector C-Arm Systems," in Multislice CT (Medical Radiology / Diagnostic Imaging), 3rd ed., M. F. Reiser, C. R. Becker,
K. Nikolaou, and G. Glazer, Eds. Springer Berlin / Heidelberg, January 2009 , ch. 3, pp. 33-51.

[46] Y. Ma, A. King, N. Gogin, C. Rinaldi, J. Gill, R. Razavi, and K. Rhode, "Real-Time Respiratory Motion Correction for Cardiac Electrophysiology Procedures Using Image-Based Coronary Sinus Catheter Tracking," in 13th International Conference on Medical Image Computing and Computer-Assisted Intervention (MICCAI) 2010, Bejing, China, ser. Lect. Notes Comput. Sci. Springer Berlin / Heidelberg, 2010, vol. 6361, pp. 391-399.

[47] A. King, R. Boubertakh, K. Rhode, Y. Ma, P. Chinchapatnam, G. Gao, T. Tangcharoen, M. Ginks, M. Cooklin, J. Gill, D. Hawkes, R. Razavi, and T. Schaeffter, "A subject-specific technique for respiratory motion correction in image-guided cardiac catheterisation procedures," Medical Image Analysis, vol. 13, no. 3, pp. 419 - 431, 2009

[48] L. Gepstein, G. Hayam, and S. A. Ben-Haim, "A novel method for nonfluoroscopic catheter-based electroanatomical mapping of the heart: In vitro and in vivo accuracy results," Circulation, vol. 95, no. 6, pp. 1611-1622, March 1997.

[49] Y. Ma, A. King, N. Gogin, G. Gijsbers, C. Rinaldi, J. Gill, R. Razavi, and K. Rhode, "Comparing image-based respiratory motion correction methods for anatomical roadmap guided cardiac electrophysiology procedures," in Functional Imaging and Modeling of the Heart, ser. Lecture Notes in Computer Science, D. Metaxas and L. Axel, Eds. Springer Berlin / Heidelberg, 2011, vol. 6666, pp. 55-62.

[50] Z. Tu, "Probabilistic boosting-tree: Learning discriminative models for classification, recognition, and clustering," in Proceedings of the Tenth IEEE International Conference on Computer Vision - Volume 2, ser. ICCV '05. Washington, DC, USA: IEEE Computer Society, Oct. 17 21 2005, pp. 1589-1596.

[51] L. Breiman, "Random Forests," Machine Learning, vol. 45, no. 1, pp. 5-32, October 2001.

[52] J. Stevenhagen, P. H. Van Der Voort, L. R. Dekker, R. W. Bullens, H. Van Den Bosch, and A. Meijer, "Three-dimensional ct overlay in comparison to cartomerge for pulmonary vein antrum isolation," Journal of Cardiovascular Electrophysiology, vol. 21, no. 6, pp. 634639, December 2010. 